\title{
Comparison of Efficacy of Candida Isolation from Oral Swab and Oral Rinse in HIV-infected Individuals
}

\author{
Gayathri Chandrasekar ${ }^{1}$, Santha D Arumugam², Vezhavendhan Nagaraj ${ }^{3}$, Kulandairaj Premlal $^{4}$, \\ Sivaramakrishnan Muthanandam ${ }^{5}$, Suganya Rajaram ${ }^{6}$
}

\begin{abstract}
Introduction: The major cause for morbidity and mortality in human immunodeficiency virus (HIV)-infected patient is fungal infection, which affects the quality of life. The most common fungal opportunistic infection is oral candidiasis. Almost all HIV patients are infected with Candida and $90-95 \%$ develop clinically, as the viral disease progresses. High plasma HIV RNA and low CD4 count are found to be associated with carriage of oral candidiasis in HIV patients. When HIV patients are treated with antiretroviral therapy, oropharyngeal candidiasis also subside simultaneously without specific antifungal therapy. The present study is intended to isolate Candida from oral swab and oral rinse and to find out which of these technique would demonstrate more isolation of Candida species.

Materials and methods: Samples are collected from 84 HIV seropositive patients attending tertiary care in Puducherry by using swab and rinse. Oral swabbing is done in areas with candidiasis and individuals without candidiasis. The oral rinse technique involves the patient holding $10 \mathrm{~mL}$ of sterile phosphate-buffered saline $(0.01 \mathrm{M}, \mathrm{pH} 7.2)$ in the mouth for 1 minute. Collected samples are inoculated in Sabouraud's dextrose agar (SDA) at $37^{\circ} \mathrm{C}$ for 48 hours.

Results: Out of 84 samples collected, 40 (47.6\%) were positive for Candida and 44 (52.4\%) were negative. Out of which swab isolated 30 (75\%) Candida and oral rinse $26(65 \%)$ of Candida with $p$ value 0.46.

Conclusion: In our study, oral swab yield more positive colonies compared to concentrated oral rinse; however, the $p$ value was statistically significant.

Keywords: Candida, Oral rinse, Swab.

Journal of Scientific Dentistry (2020): 10.5005/jp-journals-10083-0921
\end{abstract}

\section{INTRODUCTION}

The human immunodeficiency virus (HIV) causes the acquired immunodeficiency syndrome (AIDS) and it is characterized by suppression of the immune system. ${ }^{1}$ In all over the world, 36.7 million people are living with HIV. Advanced HIV patients are prone to malignancies and infections that are termed as opportunistic infections. ${ }^{1}$ Clinical severity of the disease and increased Candida species prevalence may be a reflection of immunological changes in HIV-positive patients. ${ }^{2}$ Oropharyngeal candidiasis, which is the first sign of HIV infection, ${ }^{3}$ develops at stage 3 and stage 4 of WHO clinical staging and gradually $90 \%$ of HIV patients develop candidiasis at some point. ${ }^{2}$ Though Candida albicans was found to be common organism, the prevalence of non-albicans has increased from $1429 \%$ and albicans has decreased from 86 to $71 \% .^{2-10}$ It has been stated that non-albicans like Candida glabrata, Candida dubliniensis, Candida parapsilosis, and Candida tropicalis are most commonly found organisms in HIV patients for the past few years. Emergence of non-albicans can also cause invasive candidiasis and increases mortality in HIV patients ${ }^{2,3}$ because of less susceptibility to common antifungal drugs. ${ }^{2}$ In recent studies, the most commonly resistant drug was fluconazole by Candida krusei and C. glabarata, which is one of the predominant species of nonalbicans and most organisms are susceptible to Amphotericin B. ${ }^{10}$ As prevalence of non-albicans increased and as they are resistant to normal antifungal drug, proper identification of species and antifungal susceptibility should be done for betterment of patients. The present study is intended to isolate the efficacy of Candida isolation from oral swab and oral rinse and to find out which of these technique would demonstrate more isolation of Candida species.
1,2,5,6 Department of Oral and Maxillofacial Pathology and Oral Microbiology, Indira Gandhi Institute of Dental Science, Sri Balaji Vidyapeeth, Puducherry, India

${ }^{3,4}$ Department of Oral Pathology, Indira Gandhi Institute of Dental Sciences, Sri Balaji Vidyapeeth, Puducherry, India

Corresponding Author: Gayathri Chandrasekar, Department of Oral and Maxillofacial Pathology and Oral Microbiology, Indira Gandhi Institute of Dental Science, Sri Balaji Vidyapeeth, Puducherry, India, Phone: +91 9003417067, e-mail: gayathri02.bds@gmail.com

How to cite this article: Chandrasekar G, Arumugam SD, Nagaraj V, Premlal K, Muthanandam S, Rajaram S. Comparison of Efficacy of Candida Isolation from Oral Swab and Oral Rinse in HIV-infected Individuals. J Sci Den 2020;10(1):1-2.

Source of support: Nil

Conflict of interest: None

\section{Materials and Methods}

Samples are collected using oral swab and oral rinse in HIV seropositive individuals attending a tertiary care hospital in Puducherry. A total of 84 samples were collected. Oral swab collected in areas with candidiasis and individuals without candidiasis. The oral rinse technique involves the patient holding $10 \mathrm{~mL}$ of sterile phosphate-buffered saline $(0.01 \mathrm{M}, \mathrm{pH} 7.2)$ in the mouth for 1 minute. The collected samples are transported to the microbiology laboratory without delay.

Collected samples are inoculated in Sabouraud's dextrose agar (SDA) at $37^{\circ} \mathrm{C}$ for 48 hours. Colonies formed in SDA are further confirmed for the yeast cell in gram stain and the germ tube test is 
Table 1: Candida isolated from oral swab and oral rinse from 84 samples

\begin{tabular}{lll}
\hline Results & Oral rinse (\%) & Swab (\%) \\
\hline Positive & $26(65)$ & $30(75)$ \\
Negative & $14(35)$ & $10(25)$ \\
Total & $40(100)$ & $40(100)$ \\
\hline
\end{tabular}

done to identify C. albicans and C. dubliniensis. Results are tabulated and statically analyzed.

\section{Results}

Table 1 shows that out of 84 samples collected 40 (47.6\%) were positive for Candida and 44 (52.4\%) were negative. Out of which swab isolated 30 (75\%) Candida and oral rinse $26(65 \%)$ of Candida with $p$ value of 0.46 .

\section{Discussion}

In HIV, patient's oral health is considered as an important guideline as it divulges the immune status of an individual. ${ }^{11}$ HIV patients are prone to $64-80 \%$ of oral disorders in which oral candidiasis occurs in $17-43 \%$ cases with HIV infection and $90 \%$ cases with AIDS. ${ }^{12}$ Candida is a polymorphic fungus, gram positive, and budding yeast cells, which produces pseudohyphae in culture and in tissues. ${ }^{5}$ As candidiasis is common commensal in the oral cavity, definite diagnosis of candidiasis is carried by invasion of the tissue by Candida. In a recent study, they stated that patients with oral candidiasis have 2.5 times greater risk of progression to AIDS than those who are not with oral candidiasis. ${ }^{13}$ Fungal infection is the first sign or symptoms of HIV disease and at times it may be the patient's chief complaint. It extends directly from the oral cavity to other sites in the host and makes immunocompromised patients more prone for transmission. ${ }^{14}$ Microbiological diagnosis is carried when clinical diagnosis requires confirmation in order to differentiate primary candidiasis. It is identified by smear, stain, $10 \% \mathrm{KOH}$, and by culturing in SDA. ${ }^{15}$ Though there are multiple techniques to isolate such as swab, rinse, smear, and imprint culture, the present study is intended to isolate the efficacy of Candida isolation from oral swab and oral rinse and to find out which of these technique would demonstrate more isolation of Candida species. Thereby, 84 HIV seropositive patients samples are collected individually from oral swab as well from oral rinse. The procedure involves gently rubbing lesional areas with sterile cotton inoculating into SDA for Candida growth at $37^{\circ} \mathrm{C}$ for 48 hours. Simultaneously, concentrated oral rinse is done by asking the patients to hold $10 \mathrm{~mL}$ of sterile buffered saline for 1 minute in the oral cavity. Followed by centrifuging, the sample is incubated for $24-48$ hours at $37^{\circ} \mathrm{C}$ in SDA and assessed for Candida colonies. In our study, oral swab yield more positive colonies compared to concentrated oral rinse; however, the $p$ value was statistically significant.

\section{Conclusion}

No single technique is enough to isolate yeast in order to generate universal comparable sampling method is standardize. Though hi-tech techniques are available, still the gold standard techniques such as oral swab and oral rinse are found to be more effective and can be followed in the routine.

\section{References}

1. Maheshwari M, Kaur R, Chadha S. Candida species prevalence profile in HIV seropositive patients from a major tertiary care hospital in New Delhi, India. J Pathogens 2016;2016:6204804.

2. Manikandan C, Amsath A. Isolation and rapid identification of Candida species from the oral cavity. J Pure Appl Zool 2013;1(2):172-177.

3. Vignesh Kanna B, Kumar A, Swapna M, Easow JM. Isolation and identification of candida species from various clinical samples in a tertiary care hospital. Int J Res Med Sci 2017;5(8):3520.

4. Khadka S, Sherchand JB, Pokhrel BM, et al. Isolation, speciation and antifungal susceptibility testing of Candida isolates from various clinical specimens at a tertiary care hospital, Nepal. BMC Res Notes 2017;10(1):218. DOI: 10.1186/s13104-017-2547-3.

5. German advisory committee blood, subgroups assessment of pathogen: Human Immunodeficiency virus. 2016 may 9: 203-222.

6. Turner BG, Summers MF. Structural biology of HIV. J Mole Bio 1999;285(1):1-32. DOI: 10.1006/jmbi.1998.2354.

7. Swanstrom R, Coffin J. HIV-1 pathogenesis: the virus. Cold Spring Harb Perspect Med 2012;2(12):a007443. DOI: 10.1101/cshperspect. a007443.

8. Naif HM. Pathogenesis of HIV infection. Infect Dis Rep 2013;5(1):e6. DOI: 10.4081/idr.2013.s1.e6.

9. Rajendran R, Sivapathasundaram B. Shafer's textbook of oral pathology. 5th ed., Elsevier; 2006.

10. Turner SA, Butler G. The Candida pathogenic species complex. cold spring harbor perspectives in medicine. 2014;4(9):a019778. DOI: 10.1101/cshperspect.a019778.

11. Frimpong P, Amponsah EK, Abebrese J, Kim SM. Oral manifestations and their correlation to baseline CD4 count of HIV/AIDS patients in Ghana. J Korean Assoc Oral and Maxillofac Surg 2017;43(1):29-36. DOI: 10.5125/jkaoms.2017.43.1.29.

12. Xavier TF, Auxilia A, Kannan M. Isolation and characterization of UTI pathogens from HIV positive patients of Karur District, Tamil Nadu, India. Int J Curr Microbio Appl Sci 2015;4(1):558-563.

13. Egusa H, Soysa NS, Ellepola AN, Yatani H, Samaranayake LP. Oral candidiasis in HIV-infected patients. Curr HIV Res 2008;6(6):485-499. DOI: 10.2174/157016208786501445.

14. Kamtane S, Subramaniam A, Suvarna P. A comparative study of oral candidal carriage and its association with CD4 count between HIV-positive and healthy individuals. J Int Assoc Provid AIDS Care 2013;12(1):39-43. DOI: 10.1177/1545109711423444.

15. Coronado-Castellote L, Jiménez-Soriano Y.Clinical and microbiological diagnosis of oral candidiasis. J Clin Experiment Dent 2013;5(5):e279. DOI: $10.4317 /$ jced.51242. 